\title{
Rough approximation models via graphs based on neighborhood systems
}

\author{
Abd El Fattah El Atik ${ }^{1} \cdot$ Ashraf Nawar $^{2} \cdot$ Mohammed Atef $^{2}$ (1)
}

Received: 6 July 2020 / Accepted: 19 October 2020/Published online: 5 November 2020

(C) Springer Nature Switzerland AG 2020

\begin{abstract}
Neighborhood systems are used to approximate graphs as finite topological structures. Throughout this article, we construct new types of eight neighborhoods for vertices of an arbitrary graph, say, $j$-adhesion neighborhoods. Both notions of Allam et al. and Yao will be extended via $j$-adhesion neighborhoods. We investigate new types of $j$-lower approximations and $j$ upper approximations for any subgraph of a given graph. Then, the accuracy of these approximations will be calculated. Moreover, a comparison between accuracy measures and boundary regions for different kinds of approximations will be discussed. To generate $j$-adhesion neighborhoods and rough sets on graphs, some algorithms will be introduced. Finally, a sample of a chemical example for Walczak will be introduced to illustrate our proposed methods.
\end{abstract}

Keywords Neighborhood system · Rough sets · Lower approximations · Upper approximations · Graphs · j-Accuracy measure

\section{Introduction}

Motivated by many analyzes requiring rough sets, the present paper aims for a new approach to the study of rough sets from the points of view of both neighborhood systems and graphs. Neighborhood systems on graphs based on rough sets are a generalization of Pawlak's rough set model.

Rough set theory was initially developed (Pawlak 1981) as a new mathematical methodology to deal with the vagueness and uncertainty in information systems. Many proposals made for generalizing and interpreting rough sets (Orlowska and Pawlak 1984; Pomykala 1987; Skowron and Stepaniuk 1996; Yao and Line 1996; Zirako 1994). Some applicable examples of real-life fields of the rough set method can be cited such as in Process Control, Economics, Medical Diagnosis, Biochemistry, Environmental Science, Biology, Chemistry, Psychology, Conflict

Mohammed Atef

matef@science.menofia.edu.eg

1 Department of Mathematics, Faculty of Science, Tanta University, Tanta, Egypt

2 Department of Mathematics and Computer Science, Faculty of Science, Menoufia University, Shebin El-Kom, Egypt
Analysis, Pharmacology, Banking, Market Research, Engineering, Speech Recognition, Material Science, Information Analysis, Data Analysis, Data Mining, Control and Linguistics and many other fields [See, (Allam et al. 2005; Benouini et al. 2020; Dong et al. 2004; Jensen and Shen 2004; Leung et al. 2006; Pal and Mitra 2004; Yao and Chen 2005; Zhao and Liu 2011; Zhan et al. 2019)]. In 1999, Yao (1999) introduced generalized rough sets through a binary relation; while, these approximations are not satisfied with Pawlak's properties that were applied on an equivalence relation. For this reason, Zhu (2007) studied rough approximations that depend on general relations. These approximations help to prove some properties that were not easy to prove in the classical case. From this time onwards, many types of approximations are investigated. In 2008, Abu-Donia (2008) discussed three types of lower approximations and upper approximations with respect to any binary relation based on the right neighborhoods. This generalization of approximations converted into two ways via a finite number of binary relations. In 2014, Abd ElMonsef et al. (2015) presented the main ideas about the concept of $j$-neighborhood systems and studied eight approaches for approximating rough sets. Many researchers studied the $j$-neighborhood systems on different spaces such as Abbas et al. (2016); Amer et al. (2017); Atef et al. 
(2020); Hosny (2018); Huang and Li (2018); Kozae et al. (2019).

Graph theory (Chartrand et al. 2016) is an important mathematical tool in diverse subjects. A graph $G=(V, E)$, is an ordered pair of different sets $(V, E)$, where $V$ is a nonempty set and $E$ is a subset of unordered pairs of $V$. The vertices and edges of a graph $G$ are the elements $V=V(G)$ and $E=E(G)$, respectively. A graph $G$ is finite (respectively, infinite) if the set $V(G)$ is finite (respectively, infinite). The degree of a vertex $u \in V(G)$ is the number of edges containing $u$. If there is no edge in a graph $G$ but contains a vertex $u$, then $u$ is called an isolated point, and so the degree of $u$ is zero. An edge that has the same vertex to end is called a loop, and the edge with a distinct end is called a link. A graph is simple if it has no loop and no pair of its links join the same pair of vertices. A graph that has no edge is called a null graph. A directed graph is a graph in which edges have a certain way. In addition, an undirected graph is a graph in which edges have no way. Many scholars work on the theory of graphs and applied it in many fields, see (Akram and Zafar 2018; Atef et al. 2020; Liu et al. 2020; Qin et al. 2018; Malik et al. 2018; Malik and Akram 2018; Mandal and Ranadive 2019; WilliamWest and Singh 2018). In 2018, Nada et al. (2018) initiated the study on topological structures via graphs based on the right neighborhoods. Recently, the neighborhood systems, rough sets on graphs are used to represent structures such as self-similar fractals (El Atik and Nasef 2020) and human heart (El Atik and Nasef 2020) which are useful in physics and medicine, respectively.

As a continuation of the development in the use of general relations, we construct in the present paper new types of $j$-adhesion neighborhoods from adjacent vertices of general graphs. Based on $j$-adhesion neighborhoods, we define $j$-lower approximations and $j$-upper approximations and the comparison between them and some other types of lower approximations and upper approximations will be discussed. In Sect. 2, we present the fundamental concepts and properties of that used in this paper. In Sect. 3, we introduce the new concepts of $j$-adhesion neighborhoods and study their basic properties and examples. The goal of Sect. 4 is to generalize some of Pawlak's properties. A comparison between the proposed method and the previous one is shown in Sect. 5. Finally, we apply the results on a sample that is deduced from a reduction by similarity (El Atik 2020) for Walczak's example in chemistry.

\section{Basic concepts and properties}

In this section, some basic notions of rough sets, graph theory, and a $j$-neighborhood system will be presented.
Definition 1 (Yao 1999) Let $R$ be a binary relation on a nonempty set $U$ and $A \subseteq U$. Lower approximations and upper approximations of $A$ are defined by

$$
\begin{aligned}
& \underline{R}(A)=\{x \in U: x R \subseteq A\}, \text { and } \\
& \bar{R}(A)=\{x \in U: x R \cap A \neq \phi\} \\
& x R=\{y \in U: x R y\} .
\end{aligned}
$$

where

The following properties of lower approximations and upper approximations for Pawlak (Pawlak 1982; Pawlak and Skowron 1994; Pawlak 1997) will be stated.

(L1) $\quad R(X) \subseteq X$.

(L2) $\quad \underline{R}(\phi)=\phi$.

(L3) $\quad \underline{R}(U)=U$.

(L4) $\underline{R}(X \cap Y)=\underline{R}(X) \cap \underline{R}(Y)$.

(L5) If $X \subseteq Y$, then $\underline{R}(X) \subseteq \underline{R}(Y)$.

(L6) $\underline{R}(X) \cup \underline{R}(Y) \subseteq \underline{R}(X \cup Y)$.

(L7) $\quad \underline{R}\left(X^{c}\right)=(\bar{R}(X))^{c}$.

(L8) $\underline{R}(\underline{R}(X))=\underline{R}(X)$.

(L9) $\underline{R}\left((\underline{R}(X))^{c}\right)=(\underline{R}(X))^{c}$.

(U1) $X \subseteq \bar{R}(X)$.

(U2) $\quad \bar{R}(\phi)=\phi$.

(U3) $\quad \bar{R}(U)=U$.

(U4) $\quad \bar{R}(X \cup Y)=\bar{R}(X) \cup \bar{R}(Y)$.

(U5) $\quad$ If $X \subseteq Y$, then $\bar{R}(X) \subseteq \bar{R}(Y)$.

(U6) $\bar{R}(X) \cap \bar{R}(Y) \supseteq \bar{R}(X \cap Y)$.

(U7) $\bar{R}\left(X^{c}\right)=(\underline{R}(X))^{c}$.

(U8) $\bar{R}(\bar{R}(X))=\bar{R}(X)$.

(U9) $\quad \bar{R}\left((\bar{R}(X))^{c}\right)=(\bar{R}(X))^{c}$.

Definition 2 (Abu-Donia and Salama 2012) Let $R$ be a binary relation on $U$ and $A \subseteq U$. Then, the following properties are held.

(1) Roughly $R$-definable, if $\underline{R}(A) \neq \phi$ and $\bar{R}(A) \neq X$;

(2) Internally $R$-undefinable, if $\underline{R}(A)=\phi$ and $\bar{R}(A) \neq X$;

(3) Externally $R$-undefinable, if $\underline{R}(A) \neq \phi \quad$ and $\bar{R}(A)=X$

(4) Totally $R$-undefinable, if $\underline{R}(A)=\phi$ and $\bar{R}(A)=X$.

Definition 3 (Nada et al. 2018) Let $G=(V(G), E(G))$ be a graph and $H$ be a subgraph of $G$. Lower approximations and upper approximations of $V(H)$ are defined by

$$
\begin{aligned}
\underline{\mathscr{R}}(V(H)) & =\{x \in V(G): x R \subseteq V(H)\}, \text { and } \\
\overline{\mathscr{R}}(V(H)) & =\{x \in V(G): x R \cap V(H) \neq \phi\} .
\end{aligned}
$$

Definition 4 (Yao 1999 and Allam et al. 2005) Let $G=$ $(V(G), E(G))$ be a graph, for each $x \in V(G)$. The $j$ neighborhood systems for $x, \forall j \in\{r, l,\langle r\rangle,\langle l\rangle$, $u, i,\langle u>,\langle i\rangle\}$ are defined by

(1) $N_{r}(x)=\{y \in V(G): x R y\}$. 
(2) $N_{l}(x)=\{y \in V(G): y R x\}$.

(3) $N_{<r>}(x)=\bigcap_{x \in N_{r}(y)} N_{r}(y)$.

(4) $N_{<l>}>(x)=\bigcap_{x \in N_{l}(y)} N_{l}(y)$.

(5) $\quad N_{u}(x)=N_{r}(x) \cup N_{l}(x)$.

(6) $\quad N_{i}(x)=N_{r}(x) \cap N_{l}(x)$.

(7) $N_{<u>}(x)=N_{<r>}(x) \cup N_{<l>}(x)$.

(8) $N_{<i>}(x)=N_{<r>}(x) \cap N_{<l>}(x)$.

\section{Rough approximation model via graphs using $j$-neighborhood systems}

In this section, we extent Definition 1 of Yao in terms of $j$ neighborhood systems which are defined in Definition 4. We first give an example to illustrate $j$-neighborhood systems from a simple graph.

Example 1 Let $G$ be a simple graph as shown in Fig. 1. $j$ neighborhood systems are defined as follows:

Take $j=\{r\} \quad$ and $\quad j \in\{l,<r>$, $<l>, u, i,\langle u>,<i>\}$, we have

(i) If $j=\{r, l, u, i\}$, then $N_{j}(a)=\{b, e\}, N_{j}(b)=$ $\{a, c, d\}, \quad N_{j}(c)=\{b, d\} \quad, N_{j}(d)=\{b, c, e\}$, $N_{j}(e)=\{a, d\}$.

(ii) If $j=\{\langle r\rangle,\langle l\rangle, \quad\langle u\rangle,\langle i\rangle\}$, then $N_{j}(a)=\{a, d\}, N_{j}(b)=\{b\}, N_{j}(c)=\{c\}, N_{j}(d)=$ $\{d\}, N_{j}(e)=\{b, e\}$.

Definition 5 Let $G=(V(G), E(G))$ be a graph and $H$ be a subgraph of $G$. Define a first type of lower approximations and upper approximations of $H$ which are denoted by $\underline{N_{j}}(V(H))$ and $\overline{N_{j}}(V(H))$, respectively.

$$
\begin{aligned}
& N_{j}(V(H))=\left\{x \in V(G): N_{j}(x) \subseteq V(H)\right\}, \text { and } \\
& \overline{\overline{N_{j}}}(V(H))=V(H) \bigcup\left\{x \in V(G): N_{j}(x) \cap V(H) \neq \phi\right\} .
\end{aligned}
$$

Remark 1 If $j=r$ in Definition 5, then we have approximations in Definition 1.

Definition 6 Let $G=(V(G), E(G))$ be a graph and $H$ be a subgraph of $G$. Define the $j$-boundary, $j$-positive, $j$-negative regions and $j$-accuracy measure of $H$ in terms of $j$-adhesion

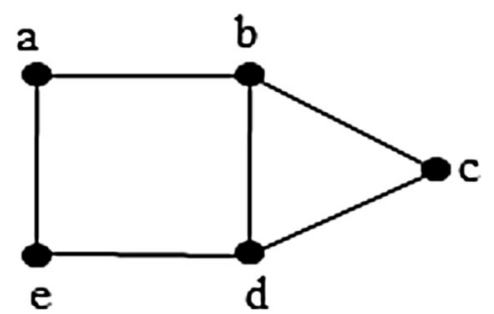

neighborhood which will be denoted by $B N D_{P_{j}}, P O S_{P_{j}}$, $N E G_{P_{j}}$ and $\alpha_{N j}$, respectively.

(1) $B N D_{N_{j}}(V(H))=\overline{N_{j}}(V(H))-N_{j}(V(H))$.

(2) $\operatorname{POS}_{N_{j}}(V(H))=N_{j}(V(H))$.

(3) $N E G_{N_{j}}(V(H))=V(G)-\overline{N_{j}}(V(H))$.

(4) $\quad \alpha_{N j}(V(H))=\frac{\left|N_{j}(V(H))\right|}{\left|\overline{N_{j}}(V(H))\right|},\left|\overline{N_{j}}(V(H))\right| \neq 0$.

Example 2 (Contine from Example 1). Take $j=r$, If $V(H)=\{b, d, e\}$, we have

(i) $\overline{N_{r}}(V(H))=V(G)$ and $\underline{N_{r}}(V(H))=\{a, c\}$.

(ii) $B N D_{N_{r}}(V(H))=\{b, d, e\}, \quad \operatorname{POS}_{N_{r}}(V(H))=$ $\{a, c\}, \quad N E G_{N_{r}}(V(H))=\{b, d, e\} \quad$ and $\quad \alpha_{N j}$ $(V(H))=\frac{2}{5}$. For $j=l,\langle r\rangle,\langle l\rangle, u, i,\langle u\rangle$, $<i>$, the results are also by the same manner.

Theorem 1 Let $G=(V(G), E(G))$ be a graph and $H$ and $K$ be subgraphs of $G$. Then, the following properties are held.

(1) $N_{j}(V(G))=V(G)$.

(2) If $V(H) \subseteq V(K)$, then $N_{j}(V(H)) \subseteq N_{j}(V(K))$.

(3) $\left.\left.\quad N_{j}(V(H) \cap V(K))=N_{j} \overline{(V}(H)\right) \cap N_{j} \overline{(V}(K)\right)$.

(4) $\bar{N}_{j}(V(H)) \cup N_{j}(V(K)) \subseteq N_{j}(V(H) \cup V(K))$.

(5) $\left.\overline{N_{j}}(V(H))=\overline{\left(N_{j}\right.}(V(H))^{c}\right)^{c}$.

(6) $\overline{\overline{N_{j}}}(\phi)=\phi$.

(7) If $V(H) \subseteq V(K)$, then $\bar{N}_{j}(V(H)) \subseteq \bar{N}_{j}(V(K))$.

(8) $\quad \overline{N_{j}}(V(H) \cup V(K))=\overline{P_{j}}(V(H)) \cup \overline{P_{j}}(V(K))$.

(9) $\quad \overline{N_{j}}(V(H) \cap V(K)) \subseteq \overline{N_{j}}(V(H)) \cap \overline{N_{j}}(V(K))$.

(10) $\overline{N_{j}}(V(H))=\left(N_{j}(V(H))^{c}\right)^{c}$.

Proof It is sufficient to prove (1), (2), (3), (4), and (5) and the other proofs are obvious.

(1) Follows from Definition 5.

(2) If $V(H) \subseteq V(K)$, then we have $N_{j}(V(H))=\{v \in$ $\left.V(G): N_{j}(v) \subseteq V(H)\right\} \subseteq \quad\left\{v \in \bar{v} V(G): N_{j}(v) \subseteq\right.$ $V(K)\}=N_{j}(V(K))$.

(3) $N_{j}(V(H \cap \bar{K}))=\left\{v \in V(G): P_{j}(v) \subseteq V(H \cap K)\right\}$. Since $V(H \cap K) \subseteq V(H)$ and $V(H \cap K) \subseteq V(K)$, then $N_{j}(v) \subseteq V(H)$ and $N_{j}(v) \subseteq V(K)$. Thus, we have $N_{j}(V(H \cap K)) \subseteq N_{j}(V(H))$ and $N_{j}(V(H \cap$ $K)) \subseteq \quad \underline{N_{j}}(V(K)) . \quad$ Therefore, $\quad N_{j}(V(H)) \cap$ $N_{j}(V(K))=\left\{v \in V(G): N_{j}(v) \subseteq V(H)\right\}$

Fig. 1 A simple graph G 


$$
\begin{aligned}
\cap & \left\{v \in V(G): N_{j}(v) \subseteq V(K)\right\} \\
& =\left\{v \in V(G): N_{j}(v) \subseteq(V(H) \cap V(K))\right\} \\
& =\left\{v \in V(G): N_{j}(v) \subseteq(V(H \cap K))\right\} \\
& =\underline{N_{j}}(V(H \cap K))=\underline{N_{j}}(V(H)) \cap \underline{N_{j}}(V(K)) .
\end{aligned}
$$

(4) The proof is similar to (3).

(5) If $v \in N_{j}(V(H))$ for every $v \in V(H)$, there exists $N_{j}(v) \subseteq V(H) . \quad$ Then, for every $v \in V(G)-[V(G)-V(H)]$, there exists $N_{j}(v)$ such that $\quad N_{j}(v) \cap[V(G)-V(H)]=\phi . \quad$ So, $v \notin \overline{N_{j}}[V(G)-V(H)]$, $v \in V(G)-\left[\overline{N_{j}}(V(G)-V(H))\right] . \quad$ Therefore, $N_{j}(V(H))=V(G)-\left[\overline{N_{j}}(V(G)-V(H))\right]=$ $\left.\overline{\left(\bar{N}_{j}\right.}(V(H))^{c}\right)^{c}$.

Example 3 (Continue for Example 1). Take $j=l$ (and also $j \in\{r,\langle r\rangle,\langle l\rangle, u, i,\langle u\rangle,\langle i>\}$ are similar).

(1) If $V(H)=\{c, d\}$, then $\bar{N}_{l}(V(H))=\{b, c, d, e\}$ and $N_{l}(V(H))=\phi$.

(2) If $V(H)=\{a\}$ and $V(K)=\{a, d\}, V(H) \subseteq V(K)$, then $\overline{N_{l}}(V(H))=\{b, e\}, \bar{N}_{l}(V(K))=\{b, c, e\}$.

(3) If $V(H)=\{a, b\}$ and $V(K)=\{a, b, d\}$, $V(H) \subseteq V(K)$, then $\underline{N}_{l}(V(H))=\phi, \quad \underline{N_{l}}(V(K))=$ $\{c, e\}$.

(4) If $V(H)=\{b\}$ and $V(K)=\{a, d\}$, then $\overline{N_{l}}(V(H \cap$ $K))=\phi$. Hence, $\bar{N}_{l}(V(H))=\{b, c, e\}$ and $\overline{N_{l}}(V(K))=\{a, b, \quad c, d, e\} . \quad$ So, $\quad \overline{P_{l}}(V(H)) \cap$ $\overline{P_{l}}(V(K))=\{b, c, e\}$. Also, $\underline{P}_{l}(V(H \cup K))=\{c, e\}$. Thus, $\underline{P}_{l}(V(H))=\phi$ and $\underline{P_{l}}(V(K))=\{e\}$. Therefore, $\underline{P_{l}}(V(H)) \cup \underline{P_{l}}(V(K)) \overline{=}\{e\}$.

Remark 2 According to Nicoletti et al. (2001); Zafar and Akram (2018), we can construct new types of rough sets, say, $j$-rough graph. So, we can also establish new $j$-approximation graphs which will be denoted by $\left(V(G), N_{j}\right), \forall$ $j \in\{r, l,\langle r\rangle,\langle l>, u, i,\langle u>,\langle i>\}$. All properties of Pawlak rough approximation can also be satisfied by the same manner.

\section{Generalized rough approximations via graphs using $j$-adhesion neighborhoods}

In this section, $j$-adhesion neighborhoods on graphs are introduced. Also, new types of $j$-lower (respectively, $j$ upper) approximations will be presented and studied.
Definition 7 Let $G=(V(G), E(G))$ be a graph. For each $x \in V(G), j$-adhesion neighborhoods are defined $\forall j \in$ $\{r, l,\langle r>,\langle l>, u, i,\langle u>,<i>\}$ as follows:

(1) $P_{r}(x)=\{y \in V(G): x R=y R\}$.

(2) $P_{l}(x)=\{y \in V(G): R x=R y\}$.

(3) $P_{<r>}(x)=\left\{y \in V(G): \bigcap_{x \in y R} y R=\bigcap_{y \in x R} x R\right\}$.

(4) $P_{<l>}(x)=\left\{y \in V(G): \bigcap_{x \in R y} R y=\bigcap_{y \in R x} R x\right\}$.

(5) $\quad P_{u}(x)=P_{r}(x) \cup P_{l}(x)$.

(6) $\quad P_{i}(x)=P_{r}(x) \cap P_{l}(x)$.

(7) $\quad P_{<u>}(x)=P_{<r>}(x) \cup P_{<l>}(x)$.

(8) $\quad P_{<i>}(x)=P_{<r>}(x) \cap P_{<l>}(x)$.

To illustrative Definition 7, we introduce Examples 4 and 5 .

Example 4 (Continue for Example 1) Take $j=$ $\left\{r, l,<r>,<l>, u, i,\langle u>,<i>\}\right.$, we have $P_{j}(a)=$ $\{a\}, P_{j}(b)=\{b\}, P_{j}(c)=\{c\}, P_{r}(d)=\{d\}, P_{r}(e)=\{e\}$.

Example 5 Let $G$ be a directed graph as shown in Fig. 2. Then, the $j$-adhesion neighborhoods are

(i) If $j \in\{r\}$, then $P_{j}(a)=\{a, d\}, P_{j}(b)=$ $\{b\}, P_{j}(c)=\{c\}, P_{j}(d)=\{a, d\}$.

(ii) If $j \in\{l,\langle r\rangle,\langle i\rangle\}$, then $P_{j}(a)=$ $\{a\}, P_{j}(b)=\{b, c\}, P_{j}(c)=\{b, c\}, P_{j}(d)=\{d\}$.

(iii) If $j \in\{i\}$, then $P_{j}(a)=\{a\}, P_{j}(b)=\{b\}, P_{j}(c)=$ $\{c\}, P_{j}(d)=\{d\}$.

(iv) If $j \in\left\{u,\langle l\rangle,\langle u>\}\right.$, then $P_{j}(a)=\{a, d\}$, $P_{j}(b)=\{b, c\}, P_{j}(c)=\{b, c\}, P_{j}(d)=\{a, d\}$.

Definition 8 Let $G=(V(G), E(G))$ be a graph and $H$ be a subgraph of $G$. The second type of lower approximations and upper approximations of $H$ which will be denoted by $\underline{P_{j}}(V(H))$ and $\overline{P_{j}}(V(H))$, respectively, is defined by

$$
\begin{aligned}
& \frac{P_{j}}{\overline{P_{j}}}(V(H))=\left\{x \in V(G): P_{j}(x) \subseteq V(H)\right\}, \text { and } \\
& =V(H) \bigcup\left\{x \in V(G): P_{j}(x) \cap V(H) \neq \phi\right\} .
\end{aligned}
$$

Definition 9 Let $G=(V(G), E(G))$ be a graph and $H$ be a subgraph of $G$. The $j$-boundary, $j$-positive, $j$-negative regions and $j$-accuracy measure of $H$ in terms of $j$-adhesion

Fig. 2 A directed graph $\mathrm{G}$

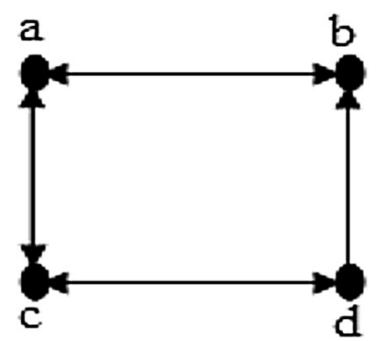


neighborhood which will be denoted by $B N D_{P_{j}}, P O S_{P_{j}}$, $N E G_{P_{j}}$ and $\alpha_{P j}$, respectively, are defined by

(i) $\quad B N D_{P_{j}}(V(H))=\overline{P_{j}}(V(H))-P_{j}(V(H))$.

(ii) $\operatorname{POS}_{P_{j}}(V(H))=\underline{P_{j}}(V(H))$.

(iii) $N E G_{P_{j}}(V(H))=V(G)-\overline{P_{j}}(V(H))$.

(iv) $\quad \alpha_{P_{j}}(V(H))=\frac{\left|P_{j}(V(H))\right|}{\left|\overline{P_{j}}(V(H))\right|},\left|\overline{P_{j}}(V(H))\right| \neq 0$.

Example 6 (Continue from Example 5). Take $j=r$. If $V(H)=\{a, c\}$, then we have

(i) $\overline{P_{r}}(V(H))=\{a, c, d\}$ and $\underline{P_{r}}(V(H))=\{c\}$.

(ii) $B N D_{P_{r}}(V(H))=\{a, d\}, \operatorname{POS}_{P_{r}}(V(H))=\{c\}$, $N E G_{P_{r}}(V(H))=\{b\}$ and $\alpha_{P j}(V(H))=\frac{1}{3}$.

For $j=l,\langle r\rangle,\langle l\rangle, u, i,\langle u\rangle,\langle i\rangle$, we have the results by similarity.

Theorem 2 Let $G=(V(G), E(G))$ be a graph and $H$ and $K$ be subgraphs of $G$. Then, the following properties are held.

(1) $P_{j}(\phi)=\phi$.

(2) $\overline{P_{j}}(V(G))=V(G)$.

(3) $\overline{P_{j}}(V(H)) \subseteq V(H)$.

(4) If $V(H) \subseteq V(K)$, then $\underline{P_{j}}(V(H)) \subseteq \underline{P_{j}}(V(K))$.

(5) $\quad P_{j}\left(P_{j}(V(H))\right)=P_{j}(V(H))$.

(6) $\overline{P_{j}}\left(\bar{V}(H) \cap V(\overline{K)})=P_{j}(V(H)) \cap P_{j}(V(K))\right.$.

(7) $\underline{P_{j}}(V(H)) \cup \underline{P_{j}}(V(K)) \subseteq \underline{P_{j}}(V(H) \cup V(K))$.

(8) $\left.\overline{P_{j}}(V(H))=\overline{\left(\overline{P_{j}}\right.}(V(H))^{c}\right)^{c}$.

(9) $\overline{\overline{P_{j}}}(\phi)=\phi$.

(10) $\overline{P_{j}}(V(G))=V(G)$.

(11) $V(H) \subseteq \overline{P_{j}}(V(H))$.

(12) If $V(H) \subseteq V(K)$, then $\overline{P_{j}}(V(H)) \subseteq \overline{P_{j}}(V(K))$.

(13) $\overline{P_{j}}\left(\overline{P_{j}}(V(H))\right)=\overline{P_{j}}(V(H))$.

(14) $\overline{P_{j}}(V(H) \cup V(K))=\overline{P_{j}}(V(H)) \cup \overline{P_{j}}(V(K))$.

(15) $\quad \overline{P_{j}}(V(H) \cap V(K)) \subseteq \overline{P_{j}}(V(H)) \cap \overline{P_{j}}(V(K))$.

(16) $\overline{P_{j}}(V(H))=\left(\underline{P_{j}}(V(H))^{c}\right)^{c}$.

Proof It is sufficient to prove properties (1), (2), (3), (4), (5), (6), (7), and (8) and other proofs are obvious.

(1) $P_{j}(\phi)=\left\{v \in V(G): P_{j}(v) \subseteq \phi\right\}=\phi$.

(2) Follows from property (1) and Definition 8.

(3) Follows from Definition 8.

(4) If $V(H) \subseteq V(K)$, then we have $P_{j}(V(H))=\{v \in$ $\left.V(G): P_{j}(v) \subseteq V(H)\right\} \subseteq\left\{v \in V(G): P_{j}(v) \subseteq\right.$ $V(K)\}=P_{j}(V(K))$.

(5) Follows from property (3) and Definition 8.
(6) $P_{j}(V(H \cap K))=\left\{v \in V(G): P_{j}(v) \subseteq V(H \cap K)\right\}$. Since $V(H \cap K) \subseteq V(H)$ and $V(H \cap K) \subseteq V(K)$, then $P_{j}(v) \subseteq V(H)$ and $P_{j}(v) \subseteq V(K)$. Thus, by property (4), we have $P_{j}(V(H \cap K)) \subseteq P_{j}(V(H))$ and $\underline{P_{j}}(V(H \cap K)) \subseteq \underline{P_{j}}(\overline{V(K)})$. Therefore, $P_{j}(V(H)) \cap$ $\overline{P_{j}}(V(K))=\left\{v \in \bar{\in}(G): P_{j}(v) \subseteq V(\bar{H})\right\} \quad \cap\{v \in$ $\left.\bar{V}(G): P_{j}(v) \subseteq V(K)\right\}=\left\{v \in V(G): P_{j}(v) \subseteq\right.$ $(V(H) \cap V(K))\}=\left\{v \in V(G): P_{j}(v) \subseteq(V(H \cap\right.$ $K))\}=\underline{P_{j}}(V(H \cap K))=\underline{P_{j}}(V(H)) \cap \underline{P_{j}}(V(K))$.

(7) The proof is similar to property (6).

(8) If $v \in P_{j}(V(H))$ for every $v \in V(H)$, there exists $P_{j}(v) \quad \subseteq V(H)$. Then, for every $v \in V(G)-[V(G)-V(H)]$, there exists $P_{j}(v)$ such that $P_{j}(v) \quad \cap[V(G)-V(H)]=\phi . \quad$ So, $\quad v \notin \overline{P_{j}}$ $[V(G)-V(H)], \quad v \in V(G)-\left[\overline{P_{j}}(V(G)-V(H))\right]$. Therefore, $P_{j}(V(H))=V(G)-\left[\overline{P_{j}}(V(G)-V(H))\right]$ $=\left(\overline{P_{j}}(V(H))^{c}\right)^{c}$.

Example 7 (Continuing from Example 5) Take $j=l$. Then

(i) If $V(H)=\{c, d\}$, then $\overline{P_{l}}(V(H))=\{b, c, d\}$ and $\underline{P_{l}}(V(H))=\{d\}$.

(ii) If $V(H)=\{a\}$ and $V(K)=\{a, b\}, V(H) \subseteq V(K)$, then $\overline{P_{l}}(V(H))=\{a\}, \overline{P_{l}}(V(K))=\{a, b, c\}$.

(iii) If $V(H)=\{a, b\}$ and $V(K)=\{a, b, d\}$, $V(H) \subseteq V(K)$, then $\quad \underline{P}_{l} \quad(V(H))=\{a\}, \quad \underline{P_{l}}$ $(V(K))=\{a, d\}$.

(iv) If $V(H)=\{b\}$ and $V(K)=\{a, c\}$, then $\overline{P_{l}}(V(H \cap$ $K))=\phi$. Hence, $\overline{P_{l}}(V(H))=\{b, c\}$ and $\overline{P_{l}}$ $(V(K))=\{a, b, c\}$. So, $\overline{P_{l}}(V(H)) \cap \overline{P_{l}}(V(K))=$ $\{b, c\}$. Also, $\underline{P_{l}}(V(H \cup K))=\{a, b, c\}$. Thus, $\underline{P_{l}}$ $(V(H))=\phi$ and $\underline{P}_{l}(V(K))=\{a\}$. Therefore, $\underline{P}_{l}(V(H)) \cup \underline{P_{l}}(V(K))=\{a\}$.

For $j=l,\langle r\rangle,\langle l\rangle, u, i,\langle u\rangle,\langle i\rangle$, we have the results by similarity .

Theorem 3 Let $G=(V(G), E(G))$ be a graph and $H, K \subseteq G$. Then, the following properties are held.

(1) $B N D_{P_{j}}(V(H))=\overline{P_{j}}(V(H)) \cap \overline{P_{j}}(V(G)-V(H))$.

(2) $B N D_{P_{j}}(V(H))=B N D_{P_{j}}(V(G)-V(H))$.

(3) $\overline{P_{j}}(V(H))=V(H) \cup B N D_{P_{j}}(V(H))$.

(4) $P_{j}(V(H))=V(H)-B N D_{P_{j}}(V(H))$.

(5) $\overline{B N} D_{P_{j}}(V(H)) \cap P_{j}(V(H))=\phi$.

(6) $B N D_{P_{j}}(V(H) \cup \overline{V(K)}) \subseteq B N D_{P_{j}}(V(H)) \cup B N D_{P_{j}}$ $(V(K))$.

(7) $B N D_{P_{j}}(V(H) \cap V(K)) \subseteq B N D_{P_{j}}(V(H)) \cup B N D_{P_{j}}$ $(V(K))$.

(8) $B N D_{P_{j}}\left(\overline{P_{j}}(V(H))\right) \subseteq B N D_{P_{j}}(V(H)$. 
(9) $B N D_{P_{j}}\left(\underline{P_{j}}(V(H))\right) \subseteq B N D_{P_{j}}(V(H)$.

(10) $B N D_{P_{j}}\left(B N D_{P_{j}}(V(H))\right) \subseteq B N D_{P_{j}}(V(H))$.

\section{Proof}

(1) $B N D_{P_{j}}(V(H))=\overline{P_{j}} \quad(V(H))-P_{j} \quad(V(H))=\overline{P_{j}}$ $(V(H)) \cap \quad\left(\underline{P_{j}} \quad(V(H))\right)^{c}=\overline{P_{j}} \quad(V(H)) \cap \quad \overline{P_{j}}$ $(V(G)-V(\bar{H}))$.

(2) $B N D_{P_{j}}(V(H))=\overline{P_{j}}(V(H)) \cap \overline{P_{j}}(V(G)-V(H))=$ $\overline{P_{j}}(V(G)-(V(G)-V(H))) \cap \overline{P_{j}}(V(G)-V(H))$ $=B N D_{P_{j}}(V(G)-V(H))$.

(3) $\quad V(H) \cup B N D_{P_{j}}(V(H))=V(H) \cup\left(\overline{P_{j}}(V(H)) \cap \overline{P_{j}}\right.$ $(V(G)-V(H)))=\left[V(H) \cup \overline{P_{j}}(V(H))\right] \cap\left[V(H) \cup \overline{P_{j}}\right.$ $(V(G)-V(H))]=\overline{P_{j}} \quad(V(H)) \quad \cap\left[V(H) \quad \cup\left(\underline{P_{j}}\right.\right.$ $\left.(V(H)))^{c}\right]=\overline{P_{j}}(V(H)) \cap V(G)=\overline{P_{j}}(V(H))$.

(4) $\quad V(H)-B N D_{P_{j}}(V(H))=V(H)-\left[\overline{P_{j}}(V(H)) \cap \overline{P_{j}}\right.$ $(V(G)-V(H))]=V(H) \cap\left[\overline{P_{j}}(V(H)) \cap \overline{P_{j}}(V(G)-\right.$ $V(H))]^{c}=V(H) \cap\left[\overline{P_{j}}(V(H))\right]^{c} \cup\left[\overline{P_{j}}(V(G)-\right.$ $V(H))]^{c}=\left[V(H) \cap P_{j}(V(G)-V(H))\right] \cup[V(H) \cap$ $\left.\underline{P_{j}}(V(H))\right]=\phi \cup \underline{P_{j}}(V(H))=\underline{P_{j}}(V(H))$.

(5) Follows from Definitions 8 and 9.

(6) $B N D_{P_{j}}(V(H) \cup V(K))=\overline{P_{j}}(V(H) \cup V(K)) \cap \overline{P_{j}}$ $(V(G)-(V(H) \cup V(K))) \subseteq\left[\overline{P_{j}}(V(H)) \cup \overline{P_{j}}(V(K))\right]$ $\cap\left[\overline{P_{j}}(V(G)-V(H)) \cap \overline{P_{j}}(V(G)-V(K))\right]=\left[\overline{P_{j}}\right.$ $\left.(V(H)) \cup \overline{P_{j}}(V(K)) \cap \overline{P_{j}} \quad(V(G)-V(H))\right] \cap\left[\overline{P_{j}}\right.$ $(V(G)-V(K))]=\left[\begin{array}{llll}\left(\overline{P_{j}}\right. & (V(H)) & \cap \overline{P_{j}} & (V(G)-\end{array}\right.$ $\left.V(H))) \cup\left(\overline{P_{j}}(V(K)) \cap \overline{P_{j}}(V(G)-V(K))\right)\right] \cap \overline{P_{j}}$ $(V(G)-V(H))=\left[\begin{array}{ll}\left(\overline{P_{j}}\right. & (V(H)) \cap \overline{P_{j}}\end{array} \quad(V(G)-\right.$ $\left.V(H))) \cap \overline{P_{j}}(V(G)-V(K))\right] \cup\left[\left(\overline{P_{j}}(V(K)) \cap \overline{P_{j}}\right.\right.$ $\left.(V(G)-V(K))) \cap \overline{P_{j}}(V(G)-V(K))\right]=\left[B N D_{P_{j}}\right.$ $\left.(V(H)) \cap \overline{P_{j}}(V(G)-V(K))\right] \cup\left[B N D_{P_{j}}(V(K)) \cap \overline{P_{j}}\right.$ $(V(G)-V(K))] \subseteq B N D_{P_{j}}(V(H)) \cup B N D_{P_{j}}(V(K))$. Therefore, $B N D_{P_{j}}(V(H) \cup V(K)) \subseteq B N D_{P_{j}}(V(H)) \cup$ $B N D_{P_{j}}(V(K))$.

(7) $B N D_{P_{j}}(V(H) \cap V(K))=\overline{P_{j}}(V(H) \cap V(K)) \cap \overline{P_{j}}$ $(V(G)-(V(H) \cap V(K))) \subseteq\left[\overline{P_{j}}(V(H)) \cap \overline{P_{j}}(V(K))\right]$ $\cap\left[\overline{P_{j}}(V(G)-V(H)) \cup \overline{P_{j}}(V(G)-V(K))\right]=\left[\overline{P_{j}}\right.$ $\left.(V(H)) \cap \overline{P_{j}}(V(K)) \cap \overline{P_{j}}(V(G)-V(H))\right] \cup\left[\overline{P_{j}}\right.$
$\left.(V(H)) \cap \quad \overline{P_{j}} \quad(V(K)) \quad \cap \overline{P_{j}} \quad(V(G)-V(K))\right]=$ $\left[B N D_{P_{j}}(V(H)) \cap \overline{P_{j}}(V(K))\right] \cup\left[B N D_{P_{j}}(V(K)) \cap \overline{P_{j}}\right.$ $(V(H))] \subseteq B N D_{P_{j}}(V(H)) \cup B N D_{P_{j}}(V(K))$.

(8) $B N D_{P_{j}}\left(\overline{P_{j}}(V(H))\right)=\overline{P_{j}}\left(\overline{P_{j}}(V(H))\right) \cap \overline{P_{j}}(V(G)$ $\left.-\overline{P_{j}}(V(H))\right)=\overline{P_{j}}(V(H)) \cap \overline{P_{j}}\left(V(G)-\overline{P_{j}} V(H)\right)$ $\subseteq \overline{P_{j}}(V(H)) \cap \overline{P_{j}}(V(G)-V(H))=B N D_{P_{j}}(V(H))$. Since $V(H) \subseteq \overline{P_{j}}(V(H))$, then $\left(\overline{P_{j}}(V(H))\right)^{c} \subseteq$ $(V(H))^{c}$ and hence $\overline{P_{j}}\left(V(G)-\overline{P_{j}}(V(H))\right) \subseteq \overline{P_{j}}$ $(V(G)-V(H))$. Thus, $B N D_{P_{j}} \quad\left(\overline{P_{j}} \quad(V(H))\right) \subseteq$ $B N D_{P_{j}}(V(H)$.

(9) $B N D_{P_{j}}\left(P_{j}(V(H))\right)=\overline{P_{j}}\left(P_{j}(V(H))\right) \cap \overline{P_{j}}(V(G)$ $\left.-P_{j}(V(\bar{H}))\right) \subseteq \overline{P_{j}}(V(H)) \cap \overline{\overline{P_{j}}}\left(V(G)-P_{j}(V(H))\right)$ $\subseteq \overline{P_{j}}(V(H)) \cap \overline{P_{j}}(V(G)-V(H))=B N \overline{D_{P_{j}}}(V(H))$. Since $\underline{P_{j}}(V(H)) \subseteq V(H)$, then $\overline{P_{j}}\left(\underline{P_{j}}(V(H))\right) \subseteq \overline{P_{j}}$ $(V(H))$. So, $B N D_{P_{j}}\left(P_{j}(V(H)) \subseteq \subseteq \overline{B N} D_{P_{j}}(V(H)\right.$.

(10) $B N D_{P_{j}}\left(B N D_{P_{j}}(V(H))\right)=B N D_{P_{j}}\left(\overline{P_{j}}(V(H)) \cap \overline{P_{j}}\right.$ $\left.\left(V(G)-P_{j}(V(H))\right)\right)=\overline{P_{j}}\left[\overline{P_{j}}(V(H)) \cap \overline{P_{j}}(V(G)\right.$ $\left.\left.-P_{j}(V(H))\right)\right] \cap \overline{P_{j}}\left[V(G)-\left(\overline{P_{j}}(V(H)) \cap \overline{P_{j}}(V(G)\right.\right.$ $\left.\left.\left.-P_{j}(V(H))\right)\right)\right] \subseteq \overline{P_{j}}\left[V(H) \cap \overline{P_{j}}(V(G)-V(H))\right] \cap$ $\overline{P_{j}}\left[\overline{P_{j}} \quad(V(H)) \cap \overline{P_{j}} \quad\left(V(G)-\overline{P_{j}} \quad(V(H))\right) \cup \quad \overline{P_{j}}\right.$ $\left.\left((V(G)) \cap \overline{P_{j}}(V(G)-V(H))\right)\right]=\overline{P_{j}}\left[\overline{P_{j}}(V(H)) \cap \overline{P_{j}}\right.$ $(V(G)-V(H))] \cap\left[\overline{P_{j}}\left(P_{j}\left(V(G)-V(H) \cup \overline{P_{j}}\left(P_{j}\right.\right.\right.\right.$ $(V(H)))))]=\overline{P_{j}}(V(H)) \cap \overline{P_{j}}(V(G)-V(H))=$ $B N D_{P_{j}}(V(H))$.

Remark 3 According to Nicoletti and Zafar results in (Nicoletti et al. 2001; Zafar and Akram 2018), new types of rough sets ,say, $j$-rough graphs and new $j$-approximation graphs, say, $\quad\left(V(G), P_{j}\right), \quad \forall \quad j \in\{r, l,<r>$, $\langle l>, u, i,\langle u\rangle,\langle i>\}$ can be constructed. Also, all Pawlak's rough approximation properties can be studied by the same manner.

In algorithm 1, we establish the system of $j$-adhesion neighborhoods from any graphs in terms of adjacent vertices and their adjacent matrix. Moreover, two vertices are neighbors if they are adjacent. 
Algorithm1. An algorithm for the $j$-adhesion neighborhoods

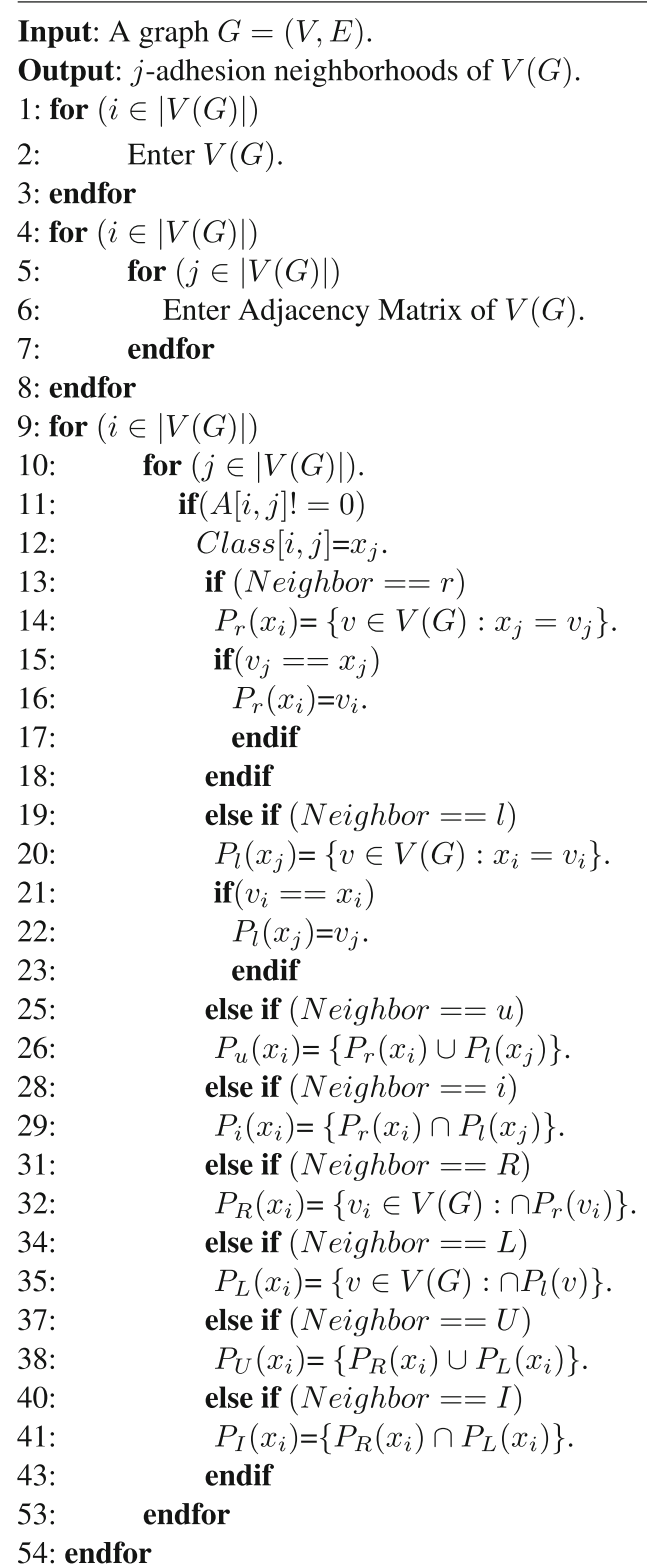

\section{Reformulation for Pawlak's properties via graphs}

In this section, we construct some of Pawlak's concepts in terms of graphs.

Definition 10 Let $G=(V(G), E(G))$ be a graph and $H$ be a subgraph of $G$. Then, we have

(i) $\quad H_{P j}$-definable $\left(H_{P j}\right.$-exact $)$ if $\overline{P_{j}}(V(H))=P_{j}(V(H))$.

(ii) $H_{P j}$-rough if $\overline{P_{j}}(V(H)) \neq P_{j}(V(H)), \forall j \in$ $\{r, l,<r>,<l>, u, i,<u>,<i>\}$.
Example 8 (Continue from Example 5) Take $j=l$. If $V(H)=\{a\}$, then $H_{P l^{-}}$-exact. While, $V(K)=\{a, b\}$ is $K_{P l^{-}}$ rough. The results for $j \in\{r,\langle r\rangle,\langle l\rangle$, $u, i,\langle u>,\langle i>\}$ are similar.

Proposition 1 Let $G=(V(G), E(G))$ be a graph. Then for all $j \in\{r,\langle r\rangle,\langle l>, u, i,\langle u\rangle,\langle i>\}$, we have

(i) Every exact graph is $H_{P j}$-exact.

(ii) Every rough graph is $H_{P j}$-rough.

Proof Obviously, by Definition 10.

Definition 11 Let $G=(V(G), E(G))$ be a graph and $H$ be a subgraph of $G$. Then, $H$ is called

(i) Roughly $H_{P j}$-definable, if $P_{j}(V(H)) \neq \phi$ and $\overline{P_{j}}$ $(V(H)) \neq V(G)$,

(ii) Internally $H_{P j}$-undefinable, if $P_{j}(V(H))=\phi$ and $\overline{P_{j}}(V(H)) \neq V(G)$,

(iii) Externally $H_{P j}$-undefinable, if $P_{j}(V(H)) \neq \phi$ and $\overline{P_{j}}(V(H))=V(G)$,

(iv) Totally $H_{P j}$-undefinable, if $P_{j}(V(H))=\phi$ and $\overline{P_{j}}(V(H))=V(G)$.

Definition 12 Let $G=(V(G), E(G))$ be a graph and $H$ be a subgraph of $G$. A membership function $\epsilon_{j}$ is called a $j$-strong if $x \in P_{j}(V(H))$. It is called a $j$-weak membership if $x \in \overline{P_{j}}$ $(V(H)), \forall j \in\{r, l,\langle r\rangle,\langle l>, u, i,\langle u>,<i>\}$.

Lemma 1 Let $G=(V(G), E(G))$ be a graph and $H$ be a subgraph of $G$. Then, we have

(i) If $x \in_{j} V(H)$, then $x \in V(H)$.

(ii) If $x \in V(H)$, then $x \overline{\epsilon_{j}} V(H)$.

Proof Obviously, by Definition 12.

The inverse implication of Lemma 1 may not be true, in general.

Example 9 (Continue from Example 5) Take $j=r$. Suppose that $V(H)=\{b, c, d\}$. Then, $P_{r}(V(H))=\{b, c\}$ and $\overline{P_{r}}(V(H))=V(G)$. It is clear that $d \in V(H)$, while, $\notin_{r}$ $V(H)$ and $a \overline{\epsilon_{r}} V(H)$ and $a \notin V(H)$. We have results for $j \in\{l,\langle r\rangle,\langle l\rangle, u, i,\langle u\rangle,\langle i\rangle\}$ by the same manner.

Proposition 2 Let $G=(V(G), E(G))$ be a graph and $H$ be a subgraph of $G$. Then, the following implications are held:

(1) $x \in_{u} V(H) \Rightarrow x \in_{r} V(H) \Rightarrow x \in_{i} V(H)$

(2) $x \underline{\in}_{u} V(H) \Rightarrow x \underline{\epsilon}_{l} V(H) \Rightarrow x \in_{i} V(H)$.

(3) $x \in \in_{<u>} V(H) \Rightarrow x \in_{<r>} V(H) \Rightarrow x \in_{<i>} V(H)$.

(4) $x \underline{\in}_{<u>} V(H) \Rightarrow x \underline{\epsilon}_{<l>} V(H) \Rightarrow x \underline{\epsilon}_{<i>} V(H)$. 
(5) $x \bar{\epsilon}_{i} V(H) \Rightarrow x \bar{\epsilon}_{r} V(H) \Rightarrow x \bar{\epsilon}_{u} V(H)$.

(6) $x \bar{\epsilon}_{i} V(H) \Rightarrow x \bar{\epsilon}_{l} V(H) \Rightarrow x \bar{\epsilon}_{u} V(H)$.

(7) $x \bar{\epsilon}_{<i>} V(H) \Rightarrow x \bar{\epsilon}_{<r>} V(H) \Rightarrow x \bar{\epsilon}_{<u>} V(H)$.

(8) $x \bar{\epsilon}_{<i>} V(H) \Rightarrow x \bar{\epsilon}_{<l>} V(H) \Rightarrow x \bar{\epsilon}_{<u>} V(H)$.

The converse may not be true, in general.

Example 10 (Continue from Example 5). Suppose that $V(H)=\{a, b\}$. Then $\underline{P}_{u}(V(H))=\phi, \underline{P}_{r}(V(H))=\{b\}$, $\underline{P}_{l}(V(H))=\{a\}$ and $\underline{P}_{i}(V(H))=\{a, b\}$. Accordingly, $b \in$ $\underline{\epsilon}_{r}(V(H))$ and $a \in \underline{\epsilon}_{l}(V(H))$. But, $b \in \underline{\notin}_{u}(V(H))$ and $a \in$ $\underline{\notin}_{u}(V(H))$. Also, $a \in \underline{\epsilon}_{i}(V(H))$ and $b \in \underline{\epsilon}_{i}(V(H))$. While, $a \in \underline{\notin}_{r}(V(H))$ and $b \in \underline{\notin}_{l}(V(H))$.

According to Definitions 8 and 9, we give an algorithm 2 to determine $P_{j}(V(H)), \overline{P_{j}}(V(H)), \quad B N D_{P_{j}}(V(H))$, $\operatorname{POS}_{P_{j}}(V(H))$ and $\overline{N E} G_{P_{j}}(V(H))$.

Algorithm2. Generate approximations on graphs

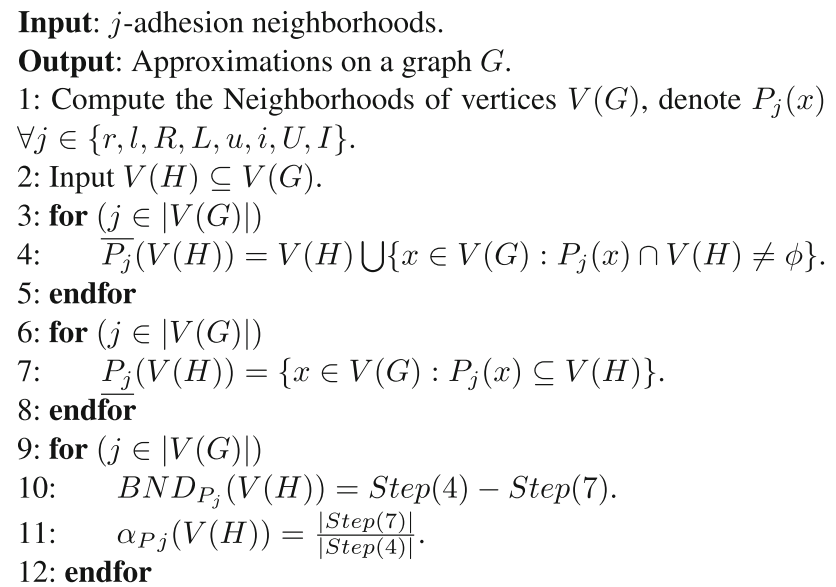

Table 1 Comparison between the boundary region and accuracy measure for Nada approachs and our study

\begin{tabular}{|c|c|c|c|c|c|c|c|c|}
\hline \multirow[t]{2}{*}{$V(H)$} & \multicolumn{4}{|c|}{$\begin{array}{l}\text { Nada's method (Nada et al. 2018) (as in Definition 1) (i.e., Definition } 5 \text { when } \\
j=j_{r} \text { ) }\end{array}$} & \multicolumn{4}{|c|}{ The current method in Definition 8} \\
\hline & $\underline{\underline{N_{r}}}(V(H))$ & $\overline{\overline{N_{r}}}(V(H))$ & $B N D_{N_{r}}(V(H))$ & $\alpha_{N_{r}}(V(H))$ & $\underline{P_{r}}(V(H))$ & $\overline{P_{r}}(V(H))$ & $B N D_{P_{r}}(V(H))$ & $\overline{\alpha_{P_{r}}(V(H))}$ \\
\hline$\{a\}$ & $\phi$ & $\{a, b, c\}$ & $\{a, b, c\}$ & 0 & $\phi$ & $\{a, d\}$ & $\{a, d\}$ & 0 \\
\hline$\{b\}$ & $\phi$ & $\{a, b, d\}$ & $\{a, b, d\}$ & 0 & $\{b\}$ & $\{b\}$ & $\phi$ & 1 \\
\hline$\{c\}$ & $\phi$ & $\{a, c, d\}$ & $\{a, c, d\}$ & 0 & $\{c\}$ & $\{c\}$ & $\phi$ & 1 \\
\hline$\{d\}$ & $\phi$ & $\{c, d\}$ & $\{c, d\}$ & 0 & $\phi$ & $\{a, d\}$ & $\{a, d\}$ & 0 \\
\hline$\{a, b\}$ & $\{b\}$ & $V(G)$ & $V(G)$ & $\frac{1}{4}$ & $\{b\}$ & $\{a, b, d\}$ & $\{a, d\}$ & $\frac{1}{3}$ \\
\hline$\{a, c\}$ & $\phi$ & $V(G)$ & $V(G)$ & 0 & $\{c\}$ & $\{a, c, d\}$ & $\{a, d\}$ & $\frac{1}{3}$ \\
\hline$\{b, d\}$ & $\phi$ & $V(G)$ & $V(G)$ & 0 & $\{b\}$ & $\{a, b, d\}$ & $\{a, d\}$ & $\frac{1}{3}$ \\
\hline$\{c, d\}$ & $\phi$ & $\{a, c, d\}$ & $\{a, c, d\}$ & 0 & $\{c\}$ & $\{a, c, d\}$ & $\{a, d\}$ & $\frac{1}{3}$ \\
\hline$\{a, b, c\}$ & $\{a, b\}$ & $V(G)$ & $\{c, d\}$ & $\frac{1}{2}$ & $\{b, c\}$ & $V(G)$ & $\{a, d\}$ & $\frac{1}{2}$ \\
\hline$\{a, c, d\}$ & $\{c\}$ & $V(G)$ & $\{a, b, d\}$ & $\frac{1}{4}$ & $\{a, c, d\}$ & $\{a, c, d\}$ & $\phi$ & 1 \\
\hline$\{b, c, d\}$ & $\{d\}$ & $V(G)$ & $\{a, b, c\}$ & $\frac{1}{4}$ & $\{b, c\}$ & $V(G)$ & $\{a, d\}$ & $\frac{1}{2}$ \\
\hline$V(G)$ & $V(G)$ & $V(G)$ & 0 & 1 & $V(G)$ & $V(G)$ & 0 & 1 \\
\hline
\end{tabular}

\section{A comparison between approach of Nada and our study}

The comparison in Table 1 between Nada's method (Nada et al. 2018) and the proposed method aims to increase the accuracy measure and reduce the boundary region by increasing lower approximations and decreasing the upper approximations. So, Example 11 will be studied at $j=r$.

Example 11 (Continue for Example 5) Lower approximations and upper approximations in Definitions 5 and 8 are given. Also, the $j$-boundary and $j$-accuracy are evaluated the comparison between them are discussed in Table 1.

In Example 12, we apply Definitions 5, 6, 8 and 9 in Walczak's example in Chemistry. We take five amino acids as a sample in Table 2. From Table 3 and Fig. 4, we show that the vertices of subgraphs $V\left(H_{1}\right)=\left\{v_{1}, v_{4}\right\}$ and $V\left(H_{2}\right)=\left\{v_{2}, v_{5}\right\}$ are necessary to determine the high energy of unfolding.

Table 2 Quantitative attributes of five amino acids

\begin{tabular}{llllll}
\hline$X$ & $a_{1}$ & $a_{2}$ & $a_{3}$ & $a_{4}$ & $a_{5}$ \\
\hline$v_{1}$ & 0.23 & 254.2 & 2.126 & 0.02 & 82.2 \\
$v_{2}$ & 0.48 & 303.6 & 2.994 & 1.24 & 112.3 \\
$v_{3}$ & 0.61 & 287.9 & 2.994 & 1.08 & 103.7 \\
$v_{4}$ & 0.45 & 282.9 & 2.933 & 0.11 & 99.1 \\
$v_{5}$ & 0.11 & 335.0 & 3.458 & 0.19 & 127.5 \\
\hline
\end{tabular}


Table 3 Comparison between boundary region and accuracy measure for Nada et al's method and our study

\begin{tabular}{|c|c|c|c|c|c|c|c|c|}
\hline \multirow[t]{2}{*}{$V(H)$} & \multicolumn{4}{|c|}{$\begin{array}{l}\text { Nada's method (Nada et al. 2018) (as in Definition 1) (i.e., Definition } 5 \text { when } \\
j=j_{r} \text { ) }\end{array}$} & \multicolumn{4}{|c|}{ Our proposed method (as in Definition 8) } \\
\hline & $\underline{N_{r}}(V(H))$ & $\overline{N_{r}}(V(H))$ & $B N D_{N_{r}}(V(H))$ & $\alpha_{N_{r}}(V(H))$ & $\underline{P_{r}}(V(H))$ & $\overline{P_{r}}(V(H))$ & $B N D_{P_{r}}(V(H))$ & $\alpha_{P_{r}}(V(H))$ \\
\hline$\left\{v_{1}\right\}$ & $\phi$ & $\left\{v_{1}\right\}$ & $\left\{v_{1}\right\}$ & 0 & $\left\{v_{1}\right\}$ & $\left\{v_{1}\right\}$ & $\phi$ & 1 \\
\hline$\left\{v_{2}\right\}$ & $\phi$ & $\left\{v_{2}\right\}$ & $\left\{v_{2}\right\}$ & 0 & $\left\{v_{2}\right\}$ & $\left\{v_{2}\right\}$ & $\phi$ & 1 \\
\hline$\left\{v_{3}\right\}$ & $\phi$ & $\left\{v_{3}\right\}$ & $\left\{v_{3}\right\}$ & 0 & $\left\{v_{3}\right\}$ & $\left\{v_{3}\right\}$ & $\phi$ & 1 \\
\hline$\left\{v_{4}\right\}$ & $\left\{v_{4}\right\}$ & $\left\{v_{1}, v_{3}, v_{4}\right\}$ & $\left\{v_{1}, v_{3}\right\}$ & $\frac{1}{3}$ & $\left\{v_{4}\right\}$ & $\left\{v_{4}\right\}$ & $\phi$ & 1 \\
\hline$\left\{v_{5}\right\}$ & $\left\{v_{5}\right\}$ & $\left\{v_{2}, v_{3}, v_{5}\right\}$ & $\left\{v_{2}, v_{3}\right\}$ & $\frac{1}{3}$ & $\left\{v_{5}\right\}$ & $\left\{v_{5}\right\}$ & $\phi$ & 1 \\
\hline$\left\{v_{1}, v_{4}\right\}$ & $\left\{v_{1}, v_{4}\right\}$ & $\left\{v_{1}, v_{3}, v_{4}\right\}$ & $\left\{v_{3}\right\}$ & $\frac{2}{3}$ & $\left\{v_{1}, v_{4}\right\}$ & $\left\{v_{1}, v_{4}\right\}$ & $\phi$ & 1 \\
\hline$\left\{v_{2}, v_{5}\right\}$ & $\left\{v_{2}, v_{5}\right\}$ & $\left\{v_{2}, v_{3}, v_{5}\right\}$ & $\left\{v_{3}\right\}$ & $\frac{2}{3}$ & $\left\{v_{2}, v_{5}\right\}$ & $\left\{v_{2}, v_{5}\right\}$ & $\phi$ & 1 \\
\hline$\left\{v_{3}, v_{4}\right\}$ & $\left\{v_{4}\right\}$ & $\left\{v_{1}, v_{2}, v_{3}, v_{4}\right\}$ & $\left\{v_{1}, v_{2}, v_{3}\right\}$ & $\frac{1}{4}$ & $\left\{v_{3}, v_{4}\right\}$ & $\left\{v_{3}, v_{4}\right\}$ & $\phi$ & 1 \\
\hline$\left\{v_{3}, v_{5}\right\}$ & $\left\{v_{5}\right\}$ & $\left\{v_{2}, v_{3}, v_{5}\right\}$ & $\left\{v_{2}, v_{3}\right\}$ & $\frac{1}{3}$ & $\left\{v_{3}, v_{5}\right\}$ & $\left\{v_{3}, v_{5}\right\}$ & $\phi$ & 1 \\
\hline$V(G)$ & $V(G)$ & $V(G)$ & $\phi$ & 1 & $V(G)$ & $V(G)$ & $\phi$ & 1 \\
\hline
\end{tabular}

Example 12 (A chemical example) Let $V(G)=$ $\left\{v_{1}, v_{2}, v_{3}, v_{4}, v_{5}\right\}$ be five amino acids (AAs) which described in terms of five attributes: $a_{1}=P I E, a_{2}=$ $S A C=$ surface area, $a_{3}=M R=$ molecular refractivity, $a_{4}=L A M=$ the side chain polarity and $a_{5}=\mathrm{Vol}=$ molecular volume (Walzak et al. 1999) as shown in Table 2. We illustrate five graphs $G_{k}$, where $k=1,2, \cdots, 5$ on $V(H) \subseteq V(G)$ in Fig. 3 such that $R_{k}=\left\{\left(x_{i}, x_{j}\right) \in\right.$ $\left.X \times X: x_{i}\left(a_{k}\right)-x_{j}\left(a_{k}\right)<\frac{\sigma_{k}}{2}, i, j, k=1,2, \cdots, 5\right\}$, where $\sigma_{k}$ represents the standard of the quantitative attributes $a_{k}$, $k=1,2,3,4,5$. From intersection of five graphs, we have a new graph $G$ in Fig. 4 which uses to construct $j$ - neighborhood systems and $j$-adhesion neighborhoods for adjacent vertices.

Take $j=r$. Then, we have

(i) $N_{r}\left(x_{1}\right)=\left\{x_{1}, x_{4}\right\}, N_{r}\left(x_{2}\right)=\left\{x_{2}, x_{5}\right\}, N_{r}\left(x_{3}\right)=$ $\left\{x_{3}, x_{4}, x_{5}\right\}, N_{r}\left(x_{4}\right)=\left\{x_{4}\right\}, N_{r}\left(x_{5}\right)=\left\{x_{5}\right\}$.

(ii) $P_{r}\left(x_{1}\right)=\left\{x_{1}\right\}, P_{r}\left(x_{2}\right)=\left\{x_{2}\right\}, P_{r}\left(x_{3}\right)=\left\{x_{3}\right\}$, $P_{r}\left(x_{4}\right)=\left\{x_{4}\right\}, P_{r}\left(x_{5}\right)=\left\{x_{5}\right\}$.

Similarily, we obtain the results of $j=\{l,<r>,<l>, u, i,<u>,<i>\}$.

Table 3 gives a comparison between the $r$-lower approximations, $r$-upper approximations, $r$-boundary regions and $r$-accuracy measures for Nada method at $j=$ $r$ and our proposed method in Definition 8 . We prove that
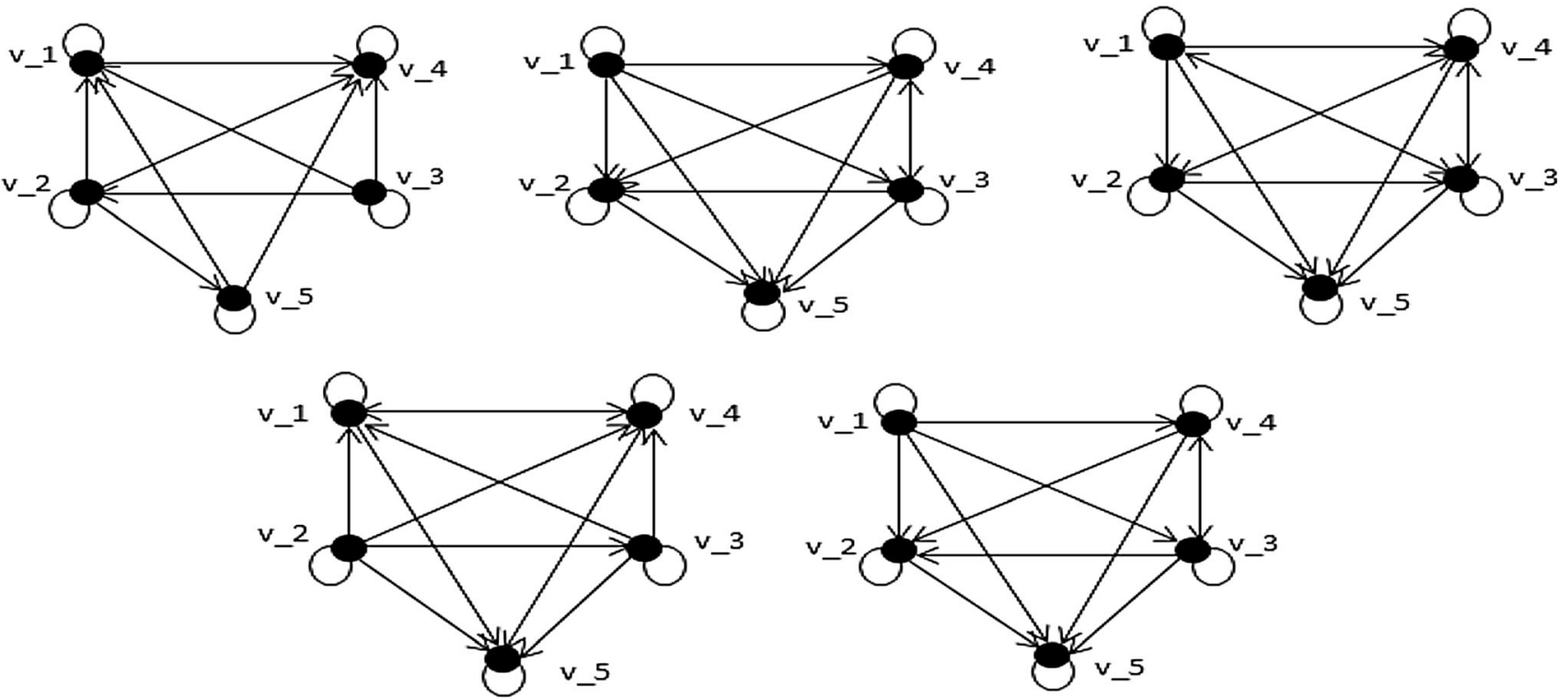

Fig. 3 A graph $G_{k}$ for $R_{k}$, where $k=1,2,3,4,5$ 


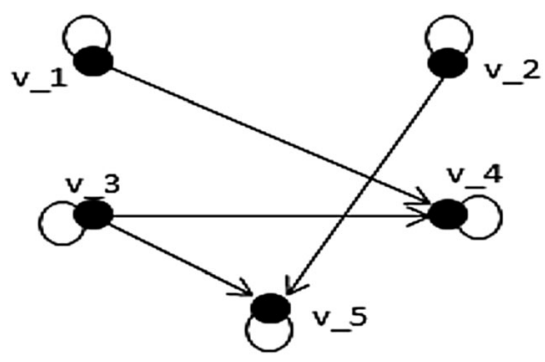

Fig. 4 Intersection of graphs $G_{k}$ to generate a graph $G$

our study method has more accurate than the previous approaches.

\section{Conclusion and future work}

$j$-Adhesion neighborhoods on general graphs are important tools to approximate graphs as finite structures. Different eight types of $j$-adhesion neighborhoods are introduced and discussed for each $j \in\{r, l, i, u,\langle r>,\langle l>,<i>,<u>\}$. By these neighborhoods, $j$-lower approximations and $j$-upper approximations will be constructed. Moreover, the relationships among $j$-approximations are superposed. Furthermore, we show that boundary regions are decreased through increasing the $j$-lower approximations and decreasing the $j$-upper approximations. So, the $j$-accuracy is more accurate than the other type defined in (Nada et al. 2018). The results in this article are very significant in decision-making, especially, to classify the family of coronavirus (Lai et al. 2020; Kampf et al. 2020) which is a topological space and type of Stone- $\breve{C}$ ech compactification.

Acknowledgements The authors would like to thank the reviewers for a careful and thorough reading of this manuscript.

Funding No applicable.

\section{Compliance with ethical standards}

Conflict of interest No potential conflict of interest was reported by the author

\section{References}

Abbas MI, Amer WS, El-Bably MK (2016) On $j$-near closure operators induced from relations and its applications. Cogent Math 3:1-17

Abd El-Monsef ME, Kozae AM, El-Bably MK (2015) Generalizing covering approximation space. J Egypt Math Soc 23:535-545
Abu-Donia HM (2008) Comparison between different kinds of approximations by using a family of binary relations. Knowl Based Syst 21:911-919

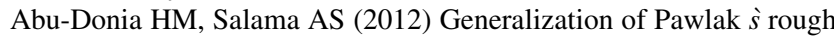
approximation spaces by using $\gamma \beta$-open sets. Int $\mathbf{J}$ Approx Reason 53:1094-1105

Allam AA, Bakeir MY, Abo-Table EA (2005) New approach for basic rough set concepts. LNCS 3641:64-73

Akram M, Zafar F (2018) Multi-criteria decision-making methods under soft rough fuzzy knowledge. J Intell Fuzzy Syst 35(3):3507-3528

Amer WS, Abbas MI, El-Bably MK (2017) On $j$-near concepts in rough sets with some applications. J Intell Fuzzy Syst 32:1089-1099

Atef M, Khalil AM, Li SG, Azzam A, El Atik AA (2020) Comparison of six types of rough approximations based on j-neighborhood space and j-adhesion neighborhood space. J Intell Fuzzy Syst 1-17 (Preprint)

Angiulli F, Pizzuti C (2005) Outlier mining in large high-dimensional data sets. IEEE Trans Knowl Data Eng 17(2):203-215

Benouini R, Batioua I, Ezghari S, Zenkouar K, Zahi A (2020) Fast feature selection algorithm for neighborhood rough set model based on Bucket and Trie structures. Granul Comput 5:329-347

Chartrand G, Lesniak L, Zhang P (2016) Textbooks in mathematics (graphs and digraphs), 6th edn. Taylor \& Francis Group, LLC

Dong G, Han J, Lam J, Pei J, Wang K, Zou W (2004) Mining constrained gradients in large databases. IEEE Trans Knowl Data Eng 16(8):922-938

El Atik A (2020) Reduction based on similarity and decision-making. J Egypt Math Soc 28(22):1-12

El Atik A, Hassan H (2020) Some nano topological structures via ideals and graphs. J Egypt Math Soc 28(41):1-21

El Atik A, Nasef A (2020) Some topological structures of fractals and their related graphs. Filomat 34(1):153-165

Hosny M (2018) On generalization of rough sets by using two different methods. J Intell Fuzzy Syst 35:979-993

Huang B, Li H (2018) Distance-based information granularity in neighborhood-based granular space. Granul Comput 3:93-110

Jensen R, Shen Q (2004) Semantics-preserving dimensionality reduction: rough and fuzzy rough based approaches. IEEE Trans Knowl Data Eng 16(12):1457-1471

Kampf G, Todt D, Pfaender S, Steinmann E (2020) Persistence of coronaviruses on inanimate surfaces and their inactivation with biocidal agents. J Hosp Infect 104:246-251

Lai CC, Shih TP, Ko WC, Tang HJ, Hsueh PR (2020) Severe acute respiratory syndrome coronavirus 2 (SARS-CoV-2) and coronavirus disease-2019 (COVID-19): the epidemic and the challenges. Int J Antimicrob Agents 55:105924

Leung Y, Wu WZ, Zhang WX (2006) Knowledge acquisition in incomplete information systems: a rough set approach. Eur J Oper Res 168:164-180

Liu K, Tsang ECC, Song J, Yu H, Chen X, Yang X (2020) Neighborhood attribute reduction approach to partially labeled data. Granul Comput 5:239-250

Kozae AM, El Atik AA, Elrokh A, Atef M (2019) New types of graphs induced by topological spaces. J Intell Fuzzy Syst 36(6):5125-5134

Malik HM, Akram M (2018) A new approach based on intuitionistic fuzzy rough graphs for decision-making. J Intell Fuzzy Syst 34(4):2325-2342

Malik HM, Akram M, Smarandache F (2018) Soft rough neutrosophic influence graphs with application. Mathematics 6(7):1-37

Mandal P, Ranadive AS (2019) Hesitant bipolar-valued fuzzy sets and bipolar-valued hesitant fuzzy sets and their applications in multiattribute group decision making. Granul Comput 4:559-583 
Nada SI, El-Atik AA, Atef M (2018) New types of topological structures via graphs. Math Methods Appl Sci 41:5801-5810

Nicoletti M, Uchôa JQ, Baptistini MT (2001) Rough relation properties. Int J Appl Math Comput Sci 11:621-635

Orlowska E, Pawlak Z (1984) Measurement and indiscernibility. Bull Pol Acad Sci Ser Sci Math 32:617-624

Pal S, Mitra P (2004) Case generation using rough sets with fuzzy representation. IEEE Trans Knowl Data Eng 16:292-300

Pawlak Z (1981) Information systems theoretical foundations. Inf Syst 6:205-218

Pawlak Z (1982) Rough sets. Int J Comput Inf Sci 11(5):341-356

Pawlak Z (1997) Rough set approach to knowledge-based decision support. Eur J Oper Res 99(1):48-57

Pawlak Z, Skowron A (1994) Rough membership function. In: Advances in the Dempster-Schafer of evidence. Wiley, New York

Pomykala JA (1987) Approximation operations in approximation space. Bull Pol Acad Sci Ser Sci Math 35:653-662

Qin J, Liu X, Martnez L (2018) Granular computing in decisionmaking. Granul Comput 3:191-192

Skowron A, Stepaniuk J (1996) Tolerance approximations spaces. Fundam Inf 27:245-253

Walczak B, Massart DL (1999) Tutorial rough set theory. Chemom Intell Lab Syst 47:1-16

William-West TO, Singh D (2018) Information granulation for rough fuzzy hypergraphs. Granul Comput 3:75-92
Yao YY (1999) On generalized rough set theory, rough sets, fuzzy sets, data mining and granular computing. In: Proc. 9th int. conf., pp 44-51

Yao YY, Chen Y (2005) Subsystem based generalizations of rough set approximations. LNCS 3488:210-218

Yao YY, Line TY (1996) Generalization of rough sets using modal logic. Int Autom Soft Comput 2:103-120

Yu Z, Bai X, Yun Z (2013) A study of rough sets based on 1neighborhood systems. Inf Sci 248:103-113

Zafar F, Akram M (2018) A novel decision-making method based on rough fuzzy information. Int J Fuzzy Syst 20(3):1000-1014

Zirako W (1994) Rough sets, fuzzy sets and knowledge discovery (RSKD1993). Workshops in computing, Springer-Verlag and British Computer Society, London, Berlin

Zhan J, Malik HM, Akram M (2019) Novel decision-making algorithms based on intuitionistic fuzzy rough environment. Int J Mach Learn Cybern 10(6):1459-1485

Zhao J, Liu L (2011) Construction of concept granule based on rough set and representation of knowledge-based complex system. Know Based Syst 24:809-815

Zhu W (2007) Generalized rough sets based on relations. Inf Sci 177:4997-5011

Publisher's Note Springer Nature remains neutral with regard to jurisdictional claims in published maps and institutional affiliations. 